\title{
Estrategias asociadas al uso de GeoGebra en un contexto de resolución de problemas
}

\author{
William Enrique Poveda Fernández ${ }^{1}$ \\ Universidad de Costa Rica \\ Daysi García-Cuéllar ${ }^{2}$ \\ Pontificia Universidad Católica del Perú
}

\begin{abstract}
RESUMEN
Este artículo presenta una experiencia en aula de matemática cuyo objetivo fue analizar cómo el uso constante de un Sistema de Geometría Dinámica (SGD), GeoGebra, en un contexto de resolución de problemas, permitió a un grupo de educadores matemáticos en formación utilizar y desarrollar estrategias de solución asociadas con el arrastre de objetos, medición de sus atributos y rastro de un punto como un medio para fomentar y desarrollar su razonamiento matemático. Los resultados indican que GeoGebra se convirtió en una herramienta que promovió nuevas formas de razonar a partir de la exploración dinámica. Las estrategias de solución permitieron a los participantes la visualización de patrones, la formulación y validación de conjeturas transitando desde el uso de argumentos visuales o empíricos hasta la construcción de un modelo algebraico o geométrico conectando conceptos de probabilidad, geometría euclidiana y de trigonometría.
\end{abstract}

Palabras clave: Resolución de Problemas; Geometría; GeoGebra; Arrastre.

\section{Strategies associated with the use of GeoGebra in a problem-solving context}

\begin{abstract}
This article presents an experience in a mathematics classroom whose objective was to analyze how the constant use of a Dynamic Geometry System (SGD), GeoGebra, in a problem-solving context, allowed a group of mathematical educators in training to use and develop strategies of solution associated with dragging objects, measuring their attributes, and tracing a point as a means of encouraging and developing their mathematical reasoning. The results indicate that GeoGebra became a tool that promoted new ways of reasoning based on dynamic exploration. The solution strategies allowed the participants to visualize patterns, formulate and validate conjectures, moving from the use of visual or empirical arguments to the construction of an algebraic or geometric model connecting concepts of probability, Euclidean geometry and trigonometry.
\end{abstract}

Keywords: Problem resolution; Geometry; GeoGebra; Dragging.

\section{Estratégias associadas ao uso do GeoGebra em um contexto de resolução de problemas}

\section{RESUMO}

Este artigo apresenta uma experiência em sala de aula de matemática cujo objetivo foi analisar como o uso constante de um Sistema de Geometria Dinâmica (SGD), GeoGebra, em um contexto de resolução de problemas, permitiu a um grupo de educadores matemáticos em formação utilizar e desenvolver estratégias de solução associada com o arrastre de objetos, medição de seus atributos e o rastro de um ponto como um médio para promover e desenvolver o raciocínio matemático. Os resultados indicam que o GeoGebra se tornou em uma ferramenta que promoveu novas formas de raciocínio baseadas na exploração dinâmica. As estratégias de solução permitiram aos participantes visualizar padrões, formular e validar conjecturas, que vão desde a utilização de argumentos visuais ou empíricos até à construção de um modelo algébrico ou geométrico ligando conceitos de

\footnotetext{
${ }^{1}$ Doctor en Ciencias en la especialidad de Matemática Educativa por el Centro de Investigación y Estudios Avanzados del Instituto Politécnico de México (CINVESTAV-IPN). Profesor-Investigador en la Universidad de Costa Rica (UCR), San Pedro de Montes de Oca, San José, Costa Rica. Endereço para correspondência: Circunvalación, San Pedro de Montes de Oca, San José, Costa Rica, CP: 11501-2060. ORCID: http://orcid.org/0000-0001-5392-0036. E-mail: william.poveda@ucr.ac.cr

${ }^{2}$ Magíster en Enseñanza de las Matemáticas por la Pontificia Universidad Católica del Perú (PUCP). Miembro del Instituto de Investigación sobre la Enseñanza de las Matemáticas (IREM-PUCP), Lima, Lima, Perú. Endereço para correspondência: Av. Universitaria 1801, San Miguel, Lima, Lima, Perú, CEP: 15088. ORCID: https://orcid.org/0000-0003-0243-6353. E-mail: garcia.daysi@pucp.pe
} 
probabilidade, geometria euclidiana e trigonometria.

Palavras-chave: Resolução de problemas; Geometria; GeoGebra; Arraste.

\section{INTRODUCCIÓN}

El uso de diferentes tecnologías digitales está presente en muchas de las actividades de la vida cotidiana; en Educación Matemática, se reconoce que es un factor importante en el desarrollo del conocimiento matemático. Con la finalidad de proveer oportunidades hacia el entendimiento y desarrollo de la matemática, NCTM (2000) propone la resolución de problemas, el uso de tecnologías digitales y la conexión entre diversas áreas de la matemática, entre otras, como medios para promover que los niños y adolescentes desarrollen formas de pensar que les permitan formular conjeturas y elaborar explicaciones para hechos numéricos o geométricos.

Durante el proceso de resolución de problemas, el uso de tecnologías digitales permite simular situaciones y reorganizar las demandas cognitivas que plantea un problema, favorece la exploración de la tarea matemática y posibilita la creación de rutas y estrategias para abordar el problema de una forma matemática (NCTM, 2009; PINHO y MORETTI, 2018). Por ejemplo, un Sistema de Geometría Dinámica (SGD), se puede utilizar para introducir nuevas clases de situaciones en la resolución de problemas matemáticos tales como formas y procedimientos de construcción, argumentación y justificación que fomentan las habilidades y contribuyen al entendimiento matemático (LESH, ZAWOJEWSKI, 2007; LEUNG, BACCAGLINI-FRANK, 2017). Además, ofrece la posibilidad de examinar situaciones matemáticas desde distintas perspectivas permitiendo la creación de nuevos escenarios en donde profesores y estudiantes pueden tener nuevas formas de visualización de los conceptos y objetos de estudio, es posible identificar y explorar de una forma más precisa los elementos matemáticos que cuando se utiliza solo papel y lápiz (SANTO-TRIGO, MORENO-ARMELLA, CAMACHO-MACHÍN, 2016).

Hegedus y Moreno-Armella (2009) argumentan que la incorporación de tecnología en las actividades escolares requiere, como primer punto, que los maestros adquieran y desarrollen nuevas habilidades y destrezas para transformar gradualmente los enfoques didácticos y las maneras de resolver problemas.

[...] la representación y exploración de tareas matemáticas mediadas por tecnologías digitales presenta nuevos desafíos para los maestros que incluyen la apropiación de las herramientas de estas tecnologías para identificar y analizar cómo [...] la práctica docente se fomenta a través de su uso. (SANTOS-TRIGO, MORENO-ARMELLA, CAMACHO-MACHÍN, 2016).

Así, un desafío específico para los profesores es integrar la tecnología en sus prácticas de enseñanza, como una manera de animar a los estudiantes a explorar ideas y desarrollar conjeturas, mientras continúan ayudándoles a comprender la necesidad de las demostraciones o los contraejemplos de las conjeturas NCTM (2000).

Las reflexiones anteriores ponen de manifiesto la importancia de identificar y reflexionar sobre posibles rutas $^{3}$ que los educadores matemáticos pueden utilizar para

\footnotetext{
${ }^{3}$ En este escrito se entiende como ruta a una manera de cómo desarrollar o abordar la resolución de problemas y el uso de las tecnologías digitales.
} 
desarrollar experiencias necesarias para comprender y poner en práctica los objetivos relacionados con currículos escolares donde la resolución de problemas y uso de tecnologías digitales son un componente importante.

Es así como surge la necesidad de fomentar las habilidades y destrezas en las formas de razonamiento matemático, mediante el uso de GeoGebra en un contexto de resolución de problemas, en futuros profesores de secundaria y bachillerato, ya que son ellos quienes tendrán la responsabilidad de decidir cómo organizar y guiar el trabajo de sus estudiantes. Este escrito expone y analiza la experiencia en el aula de matemática relacionada con el trabajo de un grupo de educadores matemáticos en formación, el rol del profesor en un contexto de resolución de problemas y el uso sistemático de GeoGebra.

Los participantes fueron ocho futuros educadores matemáticos de un curso de tercer año de la carrera Bachillerato y Licenciatura en Educación Matemática de una universidad pública en Costa Rica relacionado con el uso de tecnología digital en el aula de matemática. El trabajo se realizó durante 16 sesiones, cada una de tres horas cada semana. El objetivo fue fomentar y desarrollar el uso de diversas estrategias de resolución de problemas cuando se utiliza GeoGebra como una ruta para promover los acercamientos hacia la solución de un problema y conectar conceptos de diferentes áreas de la matemática. En este documento se exponen y analizan dos sesiones del curso donde se trabajó un problema de probabilidad, los acercamientos hacia su solución se conectaron con conceptos y teoremas de la geometría euclideana.

La pregunta que guio el desarrollo del estudio fue: ¿En qué medida las diferentes estrategias asociadas al uso de GeoGebra, en un contexto de resolución de problemas, permite a un grupo de futuros educadores matemáticos explorar representaciones de un problema y conectar diversas áreas de la matemática?

\section{MARCO TEÓRICO: RESOLUCIÓN DE PROBLEMAS Y UN SISTEMA DE GEOMETRÍA DINÁMICA}

Lesh y Zawojewski (2007) definen resolución de problemas como el proceso de interpretación de una situación matemáticamente y donde intervienen ciclos iterativos tales como expresar ideas, examinar, probar y revisar las interpretaciones matemáticas, ya sea ordenando, integrando, modificando, revisando o refinando ideas o conceptos matemáticos.

Polya (1945) en sus estudios explota la inquietud que poseen las personas por descubrir y pone de manifiesto una gama de facultades inventivas para resolver problemas. Argumenta que un problema planteado a un estudiante, por elemental que sea, pone a prueba su curiosidad y lo induce a proponer diversos métodos de soluciones que muchas veces pueden ser métodos no tradicionales, las cuales ponen en juego la originalidad del estudiante que resuelve el problema por sus propios medios, experimentando cierta fascinación por el descubrimiento. La parte más importante de la forma de razonar en matemática es la actitud relacionada con de la manera de tratar los problemas. Es decir, cuando se observa a los estudiantes en el proceso de resolución de problemas es frecuente que la mayoría no dediquen tiempo necesario para planear un plan de resolución, se descuida la fase de revisión y una vez llegado a algún resultado consideran que han terminado y no corrigen o mejoran el proceso implementado. El papel del 
profesor debe ser animar el trabajo de sus estudiantes, sugiriendo particularizaciones y generalizaciones tratando que el estudiante dé lo mejor de sí.

Schoenfeld (1985) realizó diversas investigaciones con estudiantes y profesores de matemática en las que les proponía resolver problemas bajo las premisas que los estudiantes poseían suficientes conocimientos matemáticos y los profesores, la formación para enseñar en un contexto de resolución de problemas. Los problemas propuestos tenían la particularidad de no poseer una solución inmediata por medio de la aplicación de un algoritmo. Las conclusiones de sus estudios argumentan que cuando se tiene o se quiere trabajar con resolución de problemas como una estrategia didáctica, el profesor debe tener en cuenta los siguientes factores: (1) Ser guía para los estudiantes, es decir, ayudarlos a desarrollar estrategias de resolución de problemas específicas y que se relacionen de forma clara con otras clases concretas de problemas. (2) Enseñar estrategias que permitan a los estudiantes aprender cuándo pueden utilizar estrategias apropiadas y el contenido matemático en la resolución de problemas. (3) Incrementar las creencias de los estudiantes sobre la naturaleza de las matemáticas, la resolución de problemas, y sobre sus propias competencias o formas de interactuar con situaciones matemáticas.

Cuando una persona resuelve un problema se están creando habilidades y destrezas para resolver cualquier otro tipo de problema. Schoenfeld (2007) afirma que aprender a pensar matemáticamente no es solo aprender de memoria grandes cantidades de conceptos de la materia, un aspecto central en el desarrollo del razonamiento matemático de los estudiantes es que adquieran rutas, estrategias, recursos y la disposición para involucrarse en las tareas que reflejen el quehacer matemático.

El empleo de herramientas tecnológicas en un contexto de resolución de problemas puede influir en la forma en que un estudiante interactúa con los problemas. NCTM (2000) dentro de sus principios y estándares propone que la tecnología es esencial en la enseñanza y aprendizaje de la matemática; influye en las matemáticas que se enseñan y refuerza el aprendizaje de los estudiantes.

Las herramientas digitales, como el Sistema de Geometría Dinámica, se pueden usar en el diseño de tareas para cubrir un amplio espectro epistémico desde el dibujo de figuras geométricas precisas [...] hasta la exploración de nuevos teoremas geométricos y el desarrollo del discurso de argumentación. (LEUNG, BOLITEFRANT, 2015, p. 195).

El uso de recursos disponibles media en el desarrollo del pensamiento matemático; GeoGebra, se convierte en una ayuda poderosa en las actividades de aprendizaje ya que introduce nuevas formas y procedimientos de construcción de representaciones de problemas, acercando a profesores y estudiantes a entornos de exploración dinámicos, favoreciendo el desarrollo de soluciones. Durante este proceso se fomentan habilidades en la argumentación y la justificación matemática de los hechos contribuyendo al entendimiento matemático (HEGEDUS, TALL, 2016).

Asimismo, GeoGebra introduce un aspecto dinámico, dando a los estudiantes y profesores estrategias, diferentes a las utilizadas en papel y lápiz, para visualizar y explorar los problemas. Es importante recalcar que la adquisición de estrategias para resolver problemas por parte de los estudiantes, generalmente, no se da de forma individual, Gros, Kinshuk y Maina 
(2016) argumentan que ellos necesitan desarrollar y comunicar tales estrategias en colaboración constantemente con sus compañeros y profesores para refinarlas.

Para efectos de este estudio se definen las siguientes estrategias: el arrastre de elementos involucrados en la representación dinámica, la medición de atributos de objetos matemáticos (longitud, área, perímetro, medida angular, entre otros) y el rastro que deja un punto cuando se mueve.

El arrastre de objetos es un aspecto central cuando se utiliza GeoGebra, la idea central cuando se arrastra un objeto dentro de una representación dinámica, es que esta conserve las condiciones iniciales con las cuales fue construida. Geeraerts, Venant y Tanguay (2014) lo explican con el siguiente ejemplo: al trazar la recta $\overleftrightarrow{l}$ que pasa por el punto $M$ y que es perpendicular a $\overleftrightarrow{A B}$ (con $M \notin \overleftrightarrow{A B}$ ), arrastrar del punto $M$ significa que la recta $\overleftrightarrow{l}$ debe ser perpendicular a $\overleftrightarrow{A B}$ y contener a $M$ (figura 1 ), si desaparece o no es perpendicular a $\overleftrightarrow{A B}$ o no contiene a $M$ entonces no se cumple el arrastre.

Figura 1 - Arrastre de un objeto dentro de una representación dinámica

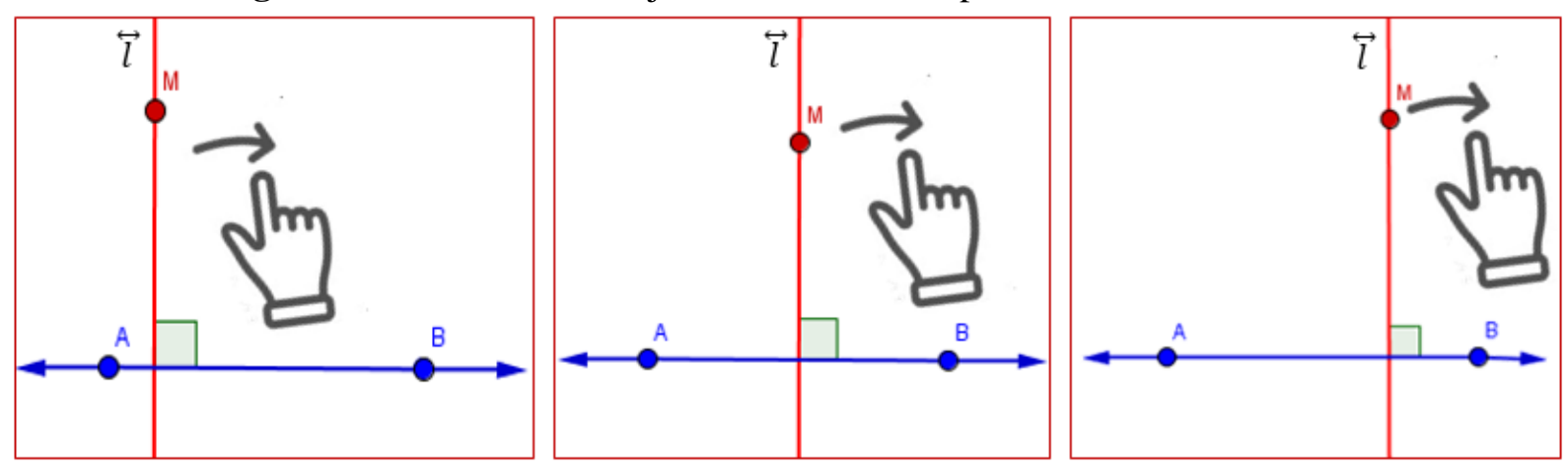

Fuente: Elaborado a partir de Geeraerts, Venant y Tanguay (2014)

Cuando una representación dinámica cumple con la prueba del arrastre, la medición de longitudes, áreas, perímetros, ángulos, pendiente de recta, entre otros, de los objetos matemáticos involucrados permite observar patrones o invariantes entre estos, lo cual se puede convertir en una ruta para establecer alguna conjetura hacia la solución del problema (HÖLZL, 2001). En Poveda (2020), se ejemplifica la estrategia de medición con el siguiente problema: dividir un cuadrado $A B C D$ en dos figuras que posean igual área. En la solución del problema, una representación dinámica en GeoGebra puede ser: construir el cuadrado $A B C D$, trazar una recta definida por un punto $P$ de $\overline{A B}$ y un punto $Q$ de $\overline{C D}(\overline{A B}$ y $\overline{C D}$ lados opuestos del cuadrado) para definir dos figuras geométricas, dado que GeoGebra proporciona el área de cada figura, al arrastrar el punto $P$ o $Q$ es posible observar cómo varían las áreas y establecer la relación de igualdad cuando la recta $\overleftrightarrow{P Q}$ pasa por el centro del cuadrado (como una posible solución general del problema). Otro aspecto importante al utilizar GeoGebra es que no se está explorando un cuadrado particular sino una familia de cuadrados, basta con arrastrar cualquiera de sus vértices para obtener otro cuadrado. Así, una conjetura basada en el arrastre y medición de áreas podría ser: la recta que pasa por el centro del cuadrado lo divide en dos figuras de igual área.

El rastro que deja un punto cuando se mueve otro, también es una estrategia importante cuando se utiliza un SGD como GeoGebra. Cuando se activa Rastro de un punto se puede 
observar la "huella" que deja de sus posiciones anteriores tras su movimiento. Esto resulta importante para visualizar, por ejemplo, la gráfica del comportamiento del área de un rectángulo en función de unos de sus lados sin la necesidad de construir un modelo algebraico (POVEDA, GOMEZ-ARCIGA, 2017).

Las estrategias anteriores asociadas al uso de GeoGebra pueden ofrecer oportunidades para explorar un problema y validar conjeturas mediante el uso de argumentos empíricos o visuales, posteriormente, se espera que las personas quien resuelven el problema construyan una demostración entrelazando o conectando diferentes conceptos y relaciones matemáticas. Durante este proceso, "al poner en relieve las conexiones matemáticas los profesores pueden contribuir a que sus estudiantes se dispongan a utilizarlas para resolver problemas, y no vean las matemáticas como un conjunto de conceptos y destrezas desconectados y aislados" (NCTM, 2000, p. 55).

Para caracterizar el proceso de resolución de problemas cuando se utiliza GeoGebra como SGD, Santos-Trigo y Camacho-Machín (2013) proponen cuatro episodios. Al primero lo llamaron comprensión del problema, el individuo que se enfrenta al problema debe identificar los objetos matemáticos presentes, definirlos y establecer sus propiedades para utilizarlos en la construcción de una representación dinámica, donde se cumpla el arrastre de objetos. El segundo es la exploración del problema, las estrategias de medición de atributos y rastro son un medio para observar patrones o invariantes entre los objetos matemáticos involucrados en el problema y que permiten formular conjeturas hacia su solución.

El tercer episodio se refiere a encontrar diferentes acercamientos hacia la solución del problema. Inicialmente, la persona que resuelve el problema puede emplear argumentos empíricos tales como datos numéricos o tablas en la hoja de cálculo en la comprobación de la conjetura y, luego, conectar diferentes conceptos y recursos matemáticos para demostrarla.

El cuarto episodio se relaciona con la integración de los diferentes tipos de soluciones del problema y sus justificaciones. Resulta importante el trabajo colaborativo, ya que los acercamientos hacia la solución del problema y las justificaciones utilizadas por una persona pueden ser conocidos por otras y, de esta manera, se pueden establecer relaciones con diferentes conceptos y relaciones matemáticos o con diferentes áreas de la matemática. Según SantosTrigo y Camacho-Machín (2013) lo anterior es un medio para desarrollar conocimiento matemático.

\section{PROCESOS METODOLÓGICOS}

En esta sección se describe el curso universitario donde se implementó el uso sistemático de GeoGebra durante el proceso de resolución de problemas matemáticos: sus objetivos, su organización, los recursos con que contaban los estudiantes y sus tiempos. En seguida, se detallan las características de sus estudiantes y la manera cómo organizaron y analizaron los datos.

El curso corresponde al tercer año de la carrera Bachillerato y Licenciatura en Educación Matemática en una universidad pública en Costa Rica. Su objetivo general es que los estudiantes reconozcan y utilicen diversas tecnologías digitales en el desarrollo de habilidades fundamentales del pensamiento matemático tales como representar un problema de diversas maneras; establecer relaciones y conexiones entre los objetos involucrados; y, formular 
conjeturas y justificarlas mediante el uso de argumentos visuales o empíricos, así como geométricos, algebraicos, entre otros.

El curso comprendió 16 semanas, una sesión de tres horas por semana, en un aula de cómputo donde cada estudiante contó con una computadora con GeoGebra. Se abordaron diversas herramientas digitales, sin embargo, se dio énfasis a GeoGebra durante la resolución de problemas relacionados con la geometría euclidiana. En este estudio se detalla y analiza el trabajo realizado en el aula de matemática realizado en las sesiones 13 y 14.

Las sesiones fueron organizadas en dos etapas, en la primera, trabajo en parejas, la idea central fue que los participantes se involucraran en el proceso de resolución de problemas compartiendo y discutiendo sus ideas con su compañero y, la segunda, trabajo plenario, consistió en conocer y analizar las propuestas de solución de otros (MASON, JOHNSTONWILDER, 2006). Para el trabajo en parejas se destinó una hora al inicio de cada sesión del curso y el resto para el trabajo plenario.

Los participantes de este estudio fueron ocho estudiantes matriculados en el curso. Sus edades estaban comprendidas entre 21 y 25 años, además todos contaban con conocimientos básicos de GeoGebra, de geometría euclidiana, de álgebra y de probabilidad básica.

El objetivo de este estudio es documentar y analizar cómo el uso sistemático de GeoGebra, en un ambiente de resolución de problemas, favorece en futuros educadores matemáticos desarrollar y utilizar estrategias asociadas con el arrastre de objetos, medición de sus atributos y rastro de un punto como un medio para conectar diversas áreas de la matemática para fomentar y desarrollar su razonamiento matemático.

\section{El problema}

Se abordó un problema con la idea de que los estudiantes conectaran la probabilidad con conceptos y relaciones de la geometría euclidiana. El problema fue el siguiente:

Sea $\triangle A B C$ un triángulo cualquiera. Sea $D$ un punto en su interior. Suponga que se mide $\angle A D B$. ¿Cuál es la probabilidad de que ese ángulo sea agudo? (Figura 2)

Figura 2 - El problema matemático del estudio

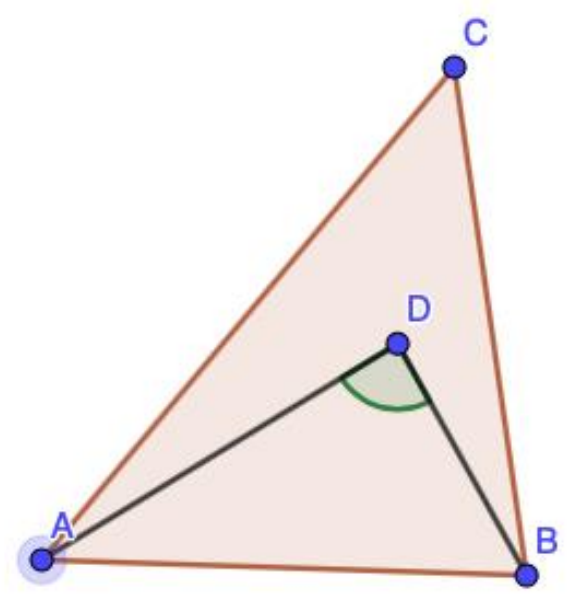

Fuente: Elaboración propia 
Los datos se recolectaron a través de archivos GeoGebra con las representaciones dinámicas que construyeron los estudiantes y sus soluciones escritas relacionadas con los episodios de la resolución del problema. También, se utilizaron las notas de campo que realizó uno de los investigadores y profesor del curso durante ambas sesiones. Los datos fueron organizados para su presentación y análisis según los episodios de resolución de problemas y uso de un SDG propuestos por Santos-Trigo y Camacho-Machín (2013).

\section{ANÁLISIS Y DISCUSIÓN DEL PROCESO DE RESOLUCIÓN DEL PROBLEMA}

En esta sección se describe el trabajo realizado en las dos sesiones del curso durante la resolución del problema propuesto y se analizan las estrategias asociadas con el uso de GeoGebra que exhibieron los estudiantes y papel que jugó el profesor. El análisis se basó en el trabajo mostrado por todo el grupo de los participantes y no de la participación y la contribución o el rendimiento de cada miembro.

\section{Episodio 1: Comprensión del problema}

En la primera fase de la resolución del problema, los ocho estudiantes representaron dinámicamente el problema, es decir, construyeron el $\triangle \mathrm{ABC}$, utilizaron la herramienta Punto en Objeto de GeoGebra para definir el punto $\mathrm{D}$ en el interior de $\triangle \mathrm{ABC}$, definieron $\angle \mathrm{ADB}$ y lo midieron. Luego, arrastraron el punto $\mathrm{D}$ para visualizar casos posibles donde el ángulo fuese agudo. Sin embargo, al arrastrar los vértices de $\triangle \mathrm{ABC}$ y observar la medida de $\angle \mathrm{ADB}$ indicaron no tener respuesta y tampoco saber cuáles son los casos favorables ni los casos totales para determinar la probabilidad utilizando la expresión matemática: $P(E)=\frac{\text { casos favorables }}{\text { casos totales }}$, donde E es el evento $\angle \mathrm{ADB}$ agudo.

El arrastre de puntos fue una estrategia que utilizaron todos los estudiantes y les permitió observar casos donde $\angle \mathrm{ADB}$ es agudo y obtuso, además, al modificar el $\triangle \mathrm{ABC}$ se percataron que para ciertos tipos de triángulos el $\angle \mathrm{ADB}$ nunca es agudo.

Las exploraciones, basadas en el arrastre del punto D y la observación de la medida del $\angle \mathrm{ADB}$, llevó a los estudiantes a pensar en casos particulares, como un primer acercamiento hacia la comprensión y solución del problema; las principales ideas, así como las representaciones que elaboraron, se muestran en el cuadro 1.

Cuadro 1 - Exploraciones en el arrastre del punto $D$ y la observación de la medida del $\angle A D B$

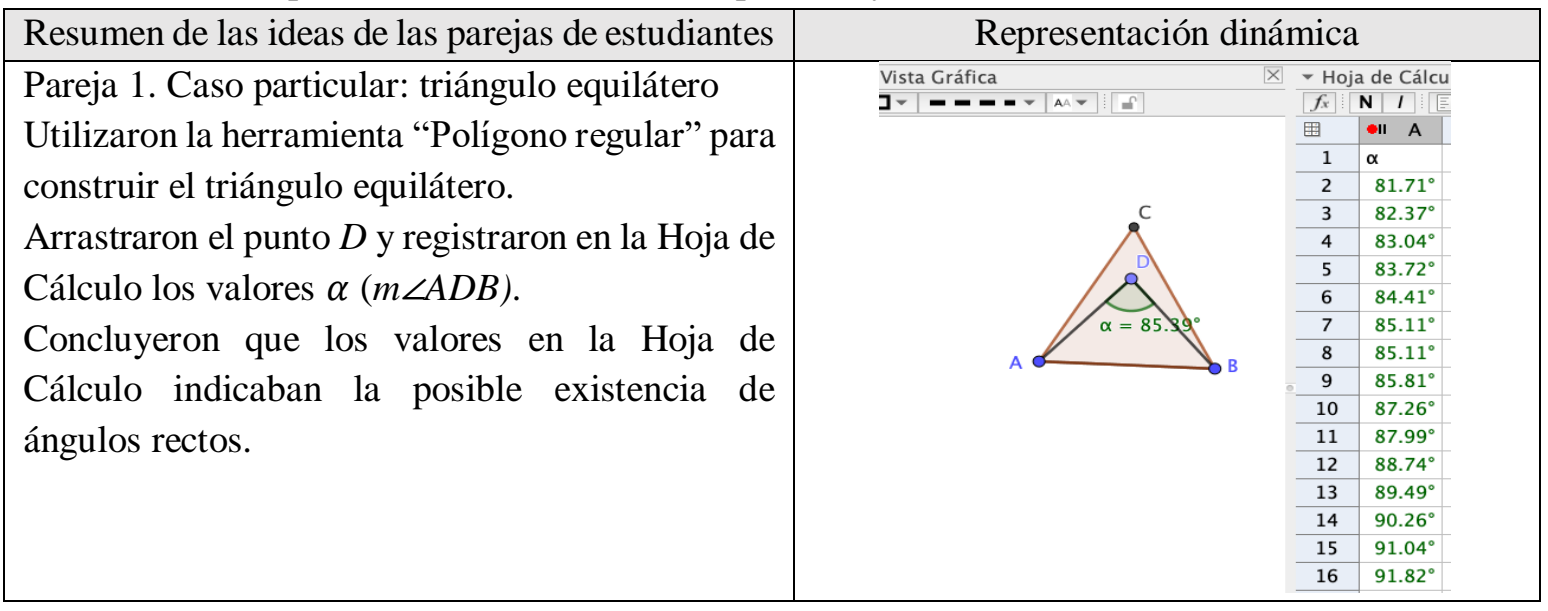




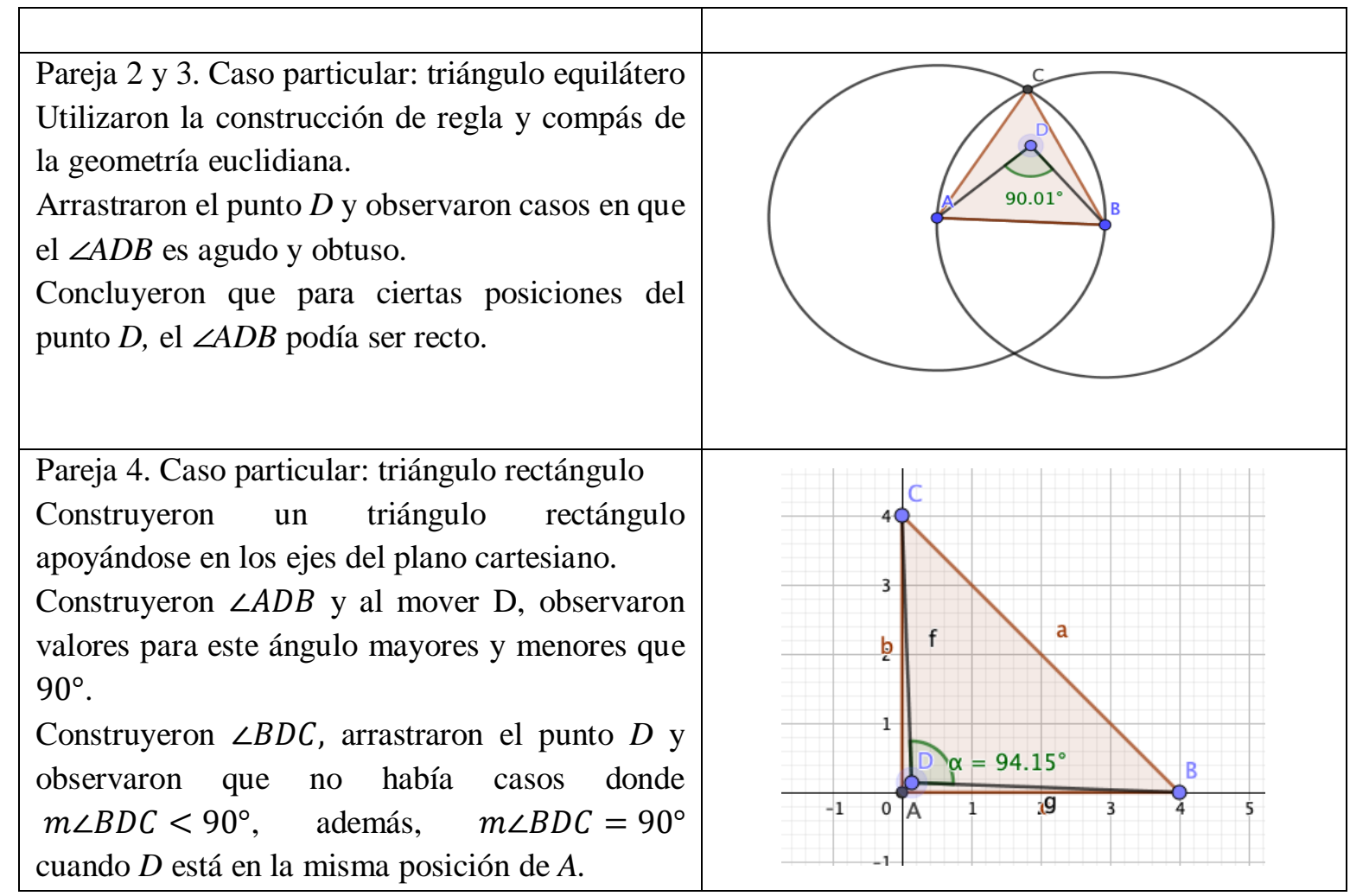

Fuente: Elaboración propia

En el trabajo plenario, la pareja 4 compartió sus hallazgos a la clase, como resultado surgió la idea de medir los ángulos internos del $\triangle A B C$ (Figura 3) para observar el comportamiento del $\angle B D C$ cuando los ángulos internos del triángulo varían. Entre todos los estudiantes exploraron los casos: $m \angle A C B<90^{\circ}$ y, luego, $m \angle A C B>90^{\circ}$. Basados en la medida $m \angle A D B$ y el arrastre del punto $D$, platearon la conjetura: Si $\triangle A B C$ es obtusángulo, con $m \angle A C B \geq 90^{\circ}$, entonces $m \angle A D B \geq 90^{\circ}$, si $m \angle A C B=90^{\circ}$ y $\mathrm{D}=\mathrm{A}$ entonces $m \angle A D B=90^{\circ}$.

Figura 3 - Representación dinámica elaborada en el trabajo plenario y una conjetura

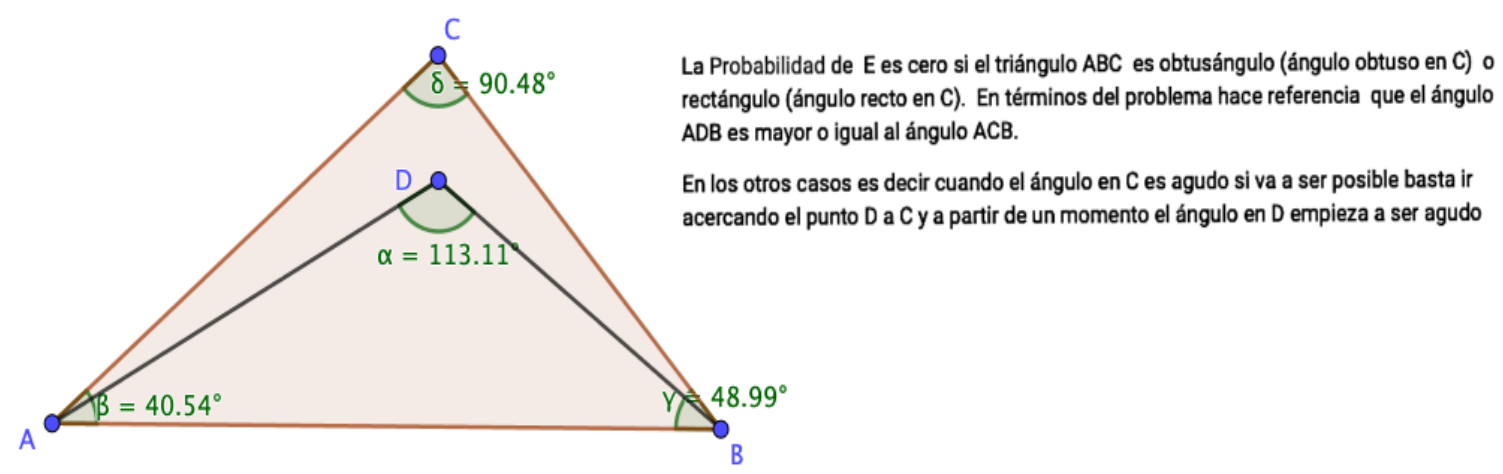

Fuente: Archivo GeoGebra elaborado en plenaria

Con la solución parcial anterior, concluyeron que $P(E)=0$ si $m \angle A C B \geq 90^{\circ}$. Luego continuaron con la exploración del caso $m \angle A C B<90^{\circ}$. En un cuestionamiento que realizó el profesor del curso, este pudo observar que los estudiantes no 
tenían claro cómo identificar los casos totales, por lo que les sugirió utilizar la estrategia de "Rastro" de GeoGebra, para observar el rastro que deja $D$ en el interior del $\triangle A B C$ (Figura 4).

Figura 4 - Representación dinámica elaborada en el trabajo plenario por la pareja 1

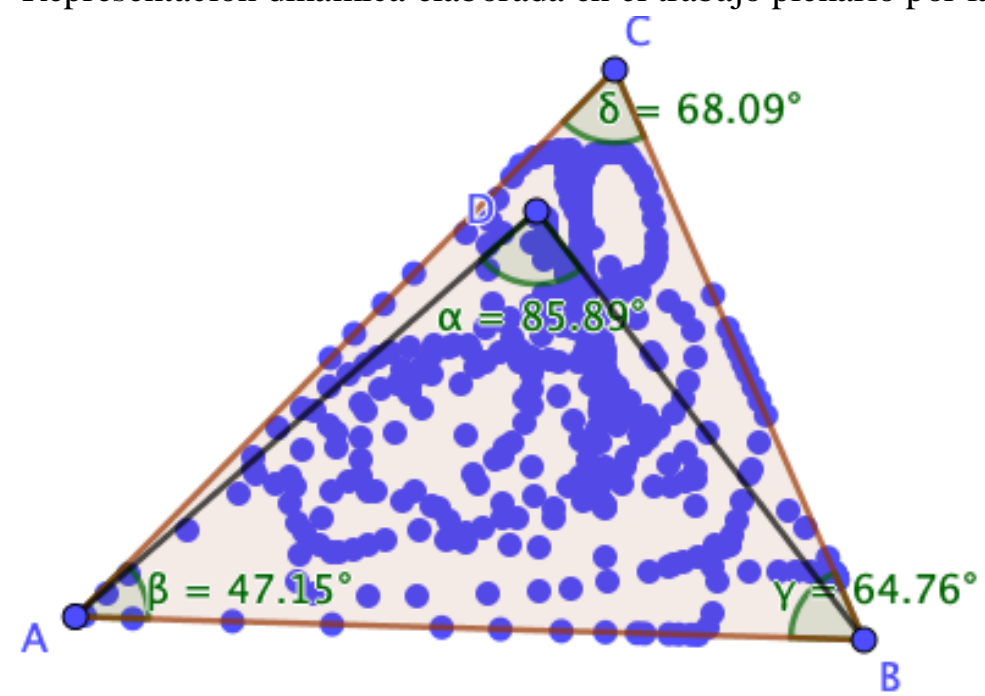

Fuente: Archivo GeoGebra elaborado por la pareja 1

Mediante el uso de la representación dinámica (Figura 4), los estudiantes asociaron el conjunto de puntos producto del arrastre y rastro de $D$ con el área del $\triangle A B C$ y por ende, con los casos totales. Luego, el profesor cuestionó ¿cómo determinar los casos favorables? Es decir ¿qué condición debe cumplir el punto $D$ para que $m \angle A D B<90^{\circ}$ ? Con esta pregunta, terminó la primera clase.

En este primer episodio, se observó que, pese a que todos los estudiantes en un trabajo en parejas lograron representar geométricamente las condiciones iniciales del problema, ninguno de ellos, en una etapa inicial, tuvo alguna idea de cómo establecer una ruta de solución. Lejos de abandonar el problema, exploraron casos particulares de triángulos gracias a las facilidades de movimiento que ofrece GeoGebra.

En el trabajo plenario, el acercamiento hacia la solución de una de las parejas fue el punto de partida para que, en plenaria, los estudiantes determinaran las condiciones necesarias para las cuales se obtiene $\angle A D B$ obtuso y así establecer las condiciones para que $P(E)=0$. En los acercamientos con el uso de GeoGebra, las estrategias de arrastre, cuantificación de las medidas de ángulos y rastro de un punto hicieron posible que los estudiantes comprendieran cómo determinar lo casos totales para ser utilizado en la expresión matemática que permite calcular la probabilidad.

\section{Episodio 2: Exploración del problema}

En la segunda clase, los estudiantes iniciaron su trabajo tratando de identificar las posiciones del punto $D$ para los cuales $m \angle A D B<90^{\circ}$ (casos favorables). El profesor introdujo la herramienta "colores dinámicos" para resaltar en color azul aquellas posiciones del punto $D$ en las cuales $m \angle A D B>90^{\circ}$ y resaltar de otro color las posiciones de $D$ en donde $m \angle A D B<$ $90^{\circ}$ (Figura 5). Por ejemplo, el profesor sugirió pintar de color verde las posiciones del punto $D$ en las cuales el $m \angle A D B<90^{\circ}$, para ello, en las propiedades del punto $D$, en el apartado de 
Avanzado, es necesario escribir en la casilla Green la instrucción $\alpha<90^{\circ}$, donde $\alpha=m \angle A D B$, en Red escribir 0 y en Blue modificar el valor a 0.5 , como se puede observar en la figura 5.

Figura 5 - Herramienta Colores dinámicos de GeoGebra

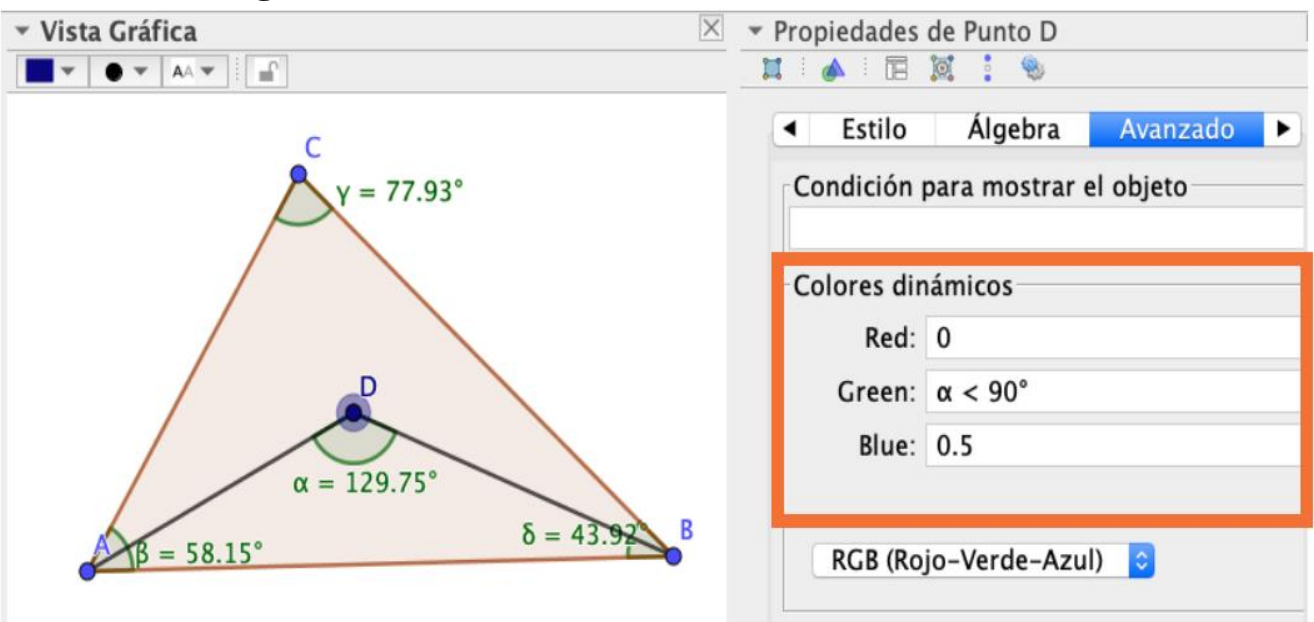

Fuente: Elaboración propia

Con el rastro del punto $D$ activado, cada pareja arrastró $D$ y centró su atención en "el borde o límite" donde cambian los colores, es decir aquellas posiciones de $D$ en las cuales $m \angle A D B$ es obtuso (color azul) o agudo (color verde), véase la Figura 6.

Figura 6 - Herramienta Colores dinámicos de GeoGebra

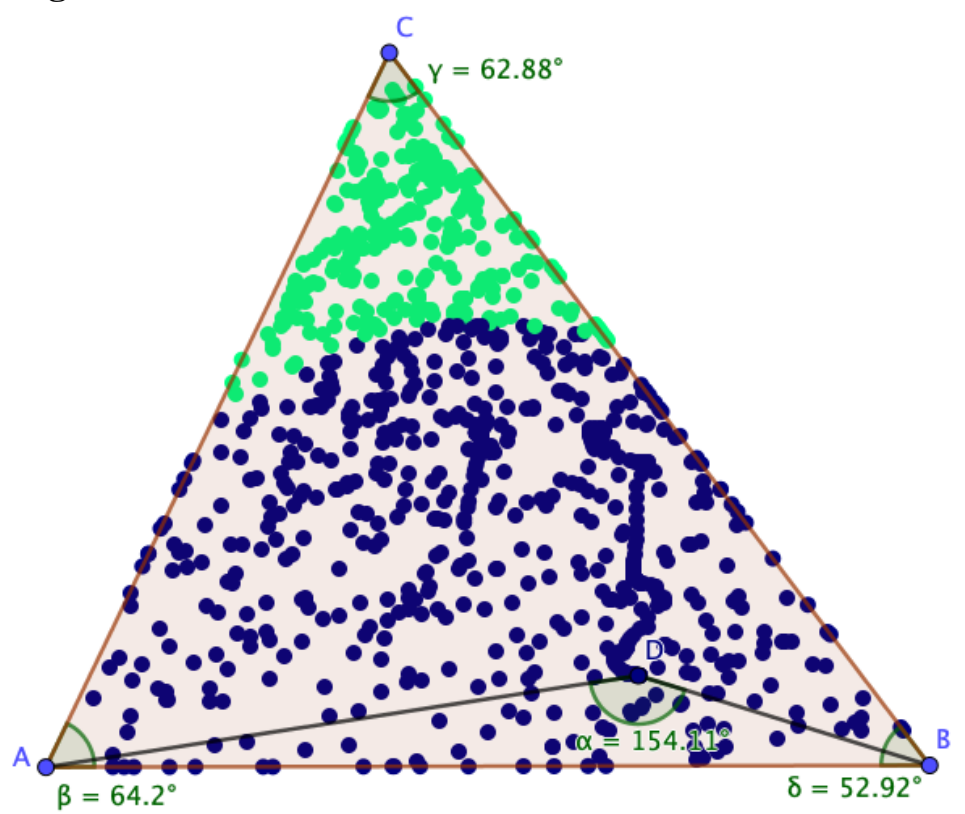

Fuente: Elaboración propia

Los colores dinámicos y el rastro del punto $D$ permitieron a los estudiantes explorar y buscar acercamientos hacia la solución del problema, la pregunta que todos se plantearon fue 
¿cómo determinar los casos favorables? En el cuadro 2 se muestran las características de las principales ideas de cada pareja de estudiantes, la conjetura que formularon y la representación dinámica que construyeron y utilizaron.

Cuadro 2 - Representación dinámica y conjeturas realizadas por las parejas de estudiantes

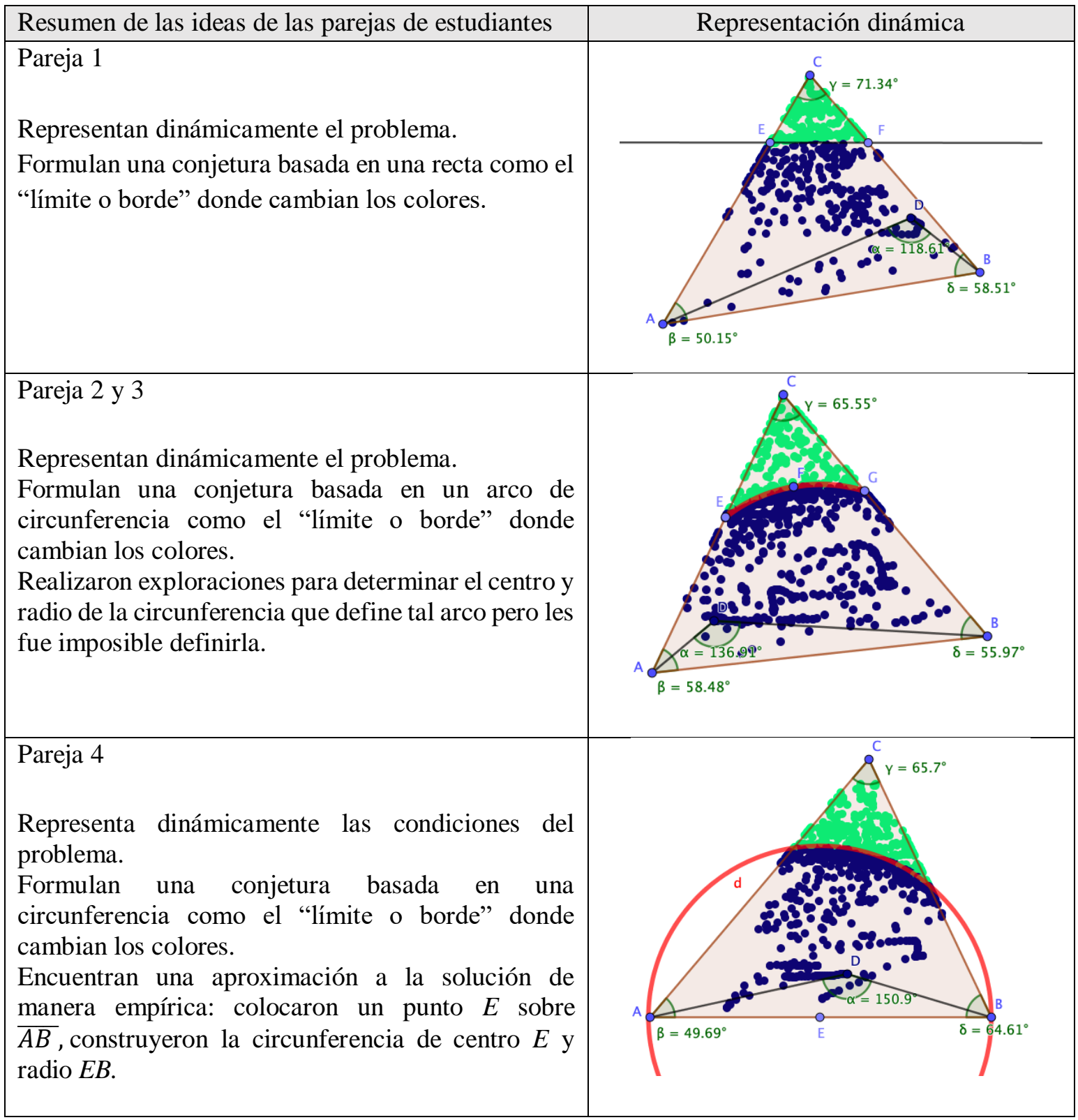

Fuente: Elaboración propia

En el trabajo plenario, la pareja 1 expuso sus ideas y los demás indicaron la falsedad de la conjetura formulada, bastó con que un estudiante les solicitara arrastrar más el punto $D$ para observar que la línea recta no es una buena aproximación para definir el "contorno o límite" donde cambia el valor del $\angle A D B$. Con respecto al trabajo de las parejas 2 y 3 , los otros estudiantes estuvieron de acuerdo con la conjetura que presentaron, sin embargo, indicaron que una limitante fue definir el centro y radio de la circunferencia que contiene tal arco. 
La pareja 4 argumentó que el "cambio de agudo a obtuso", tal como lo indicó la pareja 3, parece ser un arco de circunferencia y, al exponer su acercamiento hacia la solución, los demás comentaron que $E$, definido como el punto medio de $\overline{A B}$, podría ser el centro de la circunferencia y su radio $\frac{A B}{2}$. Para ello, arrastraron el punto $E$ y midieron las longitudes de $\overline{A E}$ y $\overline{E B}$. En la Figura 7 se muestra una captura de pantalla de la representación elaborada en la plenaria y que contiene las ideas anteriores.

Figura 7 - Captura de pantalla de la representación dinámica elaborada en plenaria y basada en las ideas de la pareja 4.

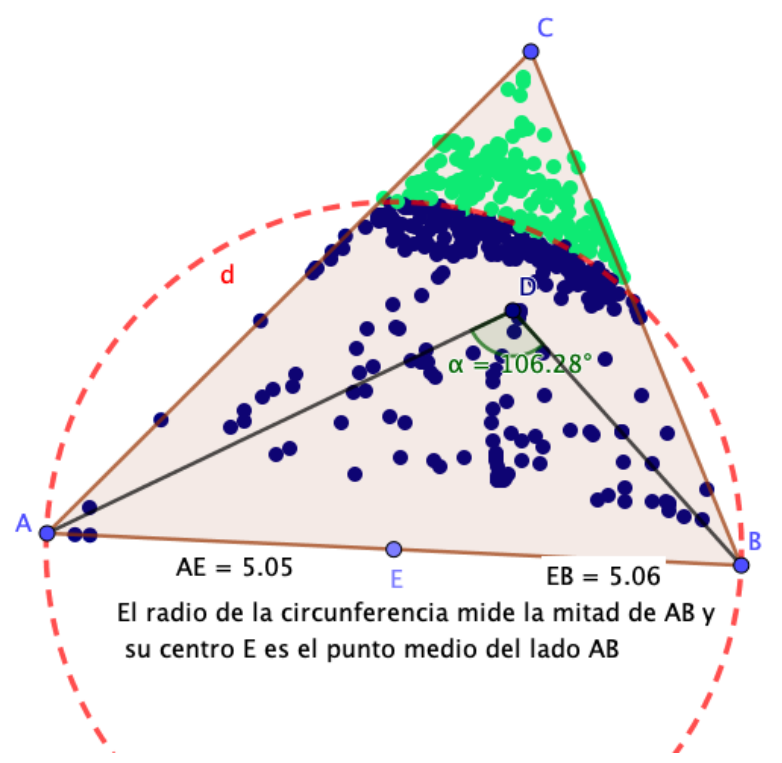

Fuente: Archivo GeoGebra elaborado en plenaria

En el segundo episodio, GeoGebra se convirtió en una herramienta importante en la visualización del comportamiento de las regiones del área que definen los casos favorables, es decir, las posiciones del punto $D$ tal que $\angle A D B$ es agudo. El resultado de arrastrar este punto y la posibilidad de observar su rastro con colores dinámicos permitió a los estudiantes establecer el límite o contorno correspondiente a la intersección de dichas regiones y asociarlas con un arco de circunferencia, exhibiendo, de esta manera, la apropiación y el entendimiento y uso de la estrategia rastro como una ruta para identificar los casos favorables de la probabilidad.

\section{Episodios 3 y 4: Búsqueda de soluciones e integración}

Una vez identificada la región asociada con los casos favorables, el cálculo la probabilidad $P(E)$ no presentó obstáculos para los estudiantes, en plenaria se discutió una forma de obtener el área asociada a los casos favorables. Para ello:

1. Utilizaron la representación dinámica que se muestra en la Figura 8, en una fase inicial, determinaron la suma de las áreas de $\triangle C E G$ y $\triangle E B F$ y del sector circular $E F G$ con ayuda de GeoGebra.

2. Calcularon $P(E)=\frac{\text { casos favorables }}{\text { casos totales }}=\frac{\text { suma de áreas ( } \triangle C E G \text { y } \triangle E B F \text { y del sector circular } E F G)}{\text { área } \triangle A B C}$, el resultado se muestra en la etiqueta "ProbabilidadAgudo" de la Figura 8. 
Figura 8 - Captura de pantalla de la representación dinámica elaborada en plenaria

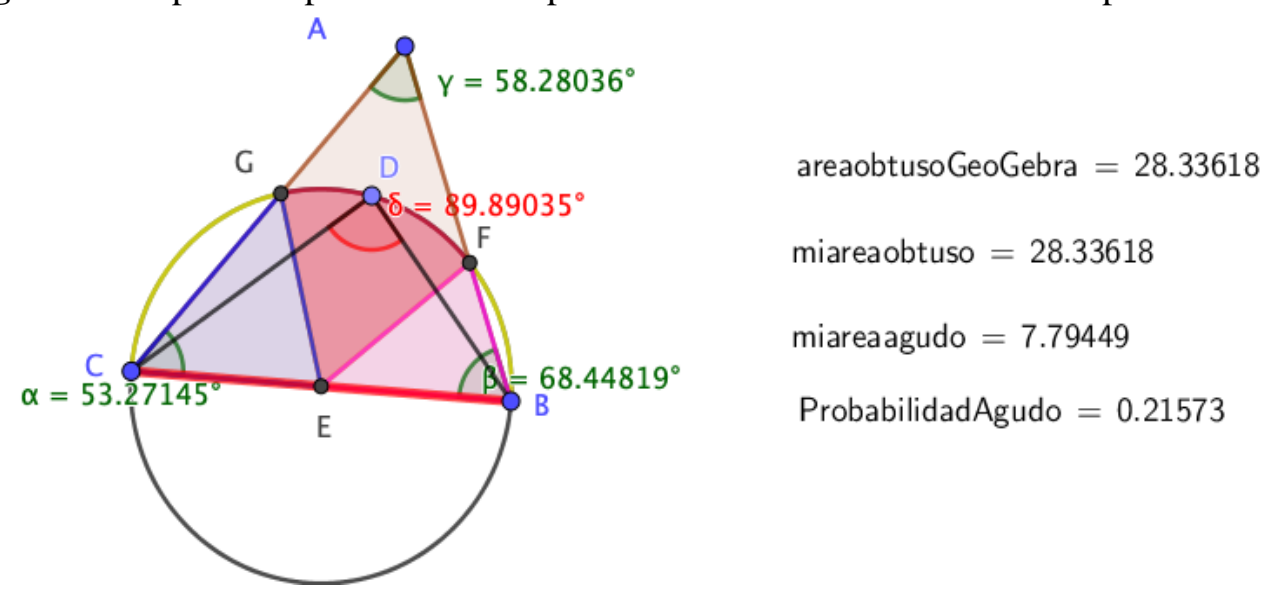

Fuente: Archivo GeoGebra elaborado en plenaria

Luego, realizaron manualmente los cálculos para determinar el área correspondiente donde el punto $D$ define un ángulo agudo (se muestra en la etiqueta "miareaagudo" de la Figura 8) y comprobaron la respuesta con la proporcionada con GeoGebra. Los cálculos realizados en papel y lápiz por la pareja 4 se muestran en la Figura 9. En la resolución del problema, inicialmente enfocado en probabilidad, los estudiantes conectaron conceptos geométricos y trigonométricos.

Figura 9 - Cálculos realizados por la pareja 4 para determinar los casos favorables.

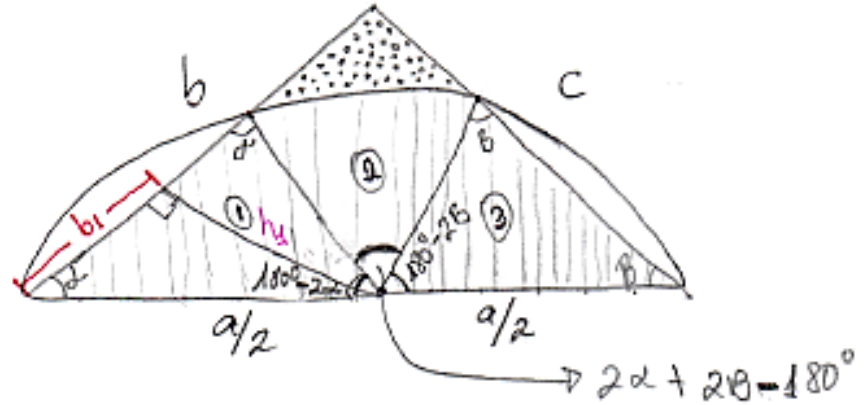

VIII : área donde el ángulo es obtuso

$$
\begin{aligned}
A_{1} & =\frac{2 b_{1} \cdot h_{1}}{2}=b_{1} \cdot h_{1}=\frac{a^{2} \operatorname{sen} \alpha \cdot \cos \alpha}{4} \\
& \cdot \operatorname{sen} \alpha=\frac{h_{1}}{a / 2} \Leftrightarrow \frac{a \cdot \operatorname{sen} \alpha}{2}=h_{1} \\
& \cdot \cos \alpha=\frac{b_{1}}{a / 2} \Leftrightarrow \frac{a \cdot \cos \alpha}{2}=b_{1}
\end{aligned}
$$

Fuente: Ficha de trabajo de la pareja 4 
Una vez que los estudiantes calcularon $P(E)$, uno de ellos cuestionó, ¿por qué el cambio de ángulos agudos y obtusos se da en tal arco de circunferencia? Entre todos enunciaron el teorema: "Si $D$ es un punto de la circunferencia de diámetro $A B$, distinto de $A$ y $B$, entonces el $\triangle A D B$ es rectángulo en $D$ ".

Cada pareja utilizó GeoGebra para representar las hipótesis del teorema, en general, construyeron una circunferencia $c$ de diámetro $A B$, colocaron un punto sobre $D$ sobre $c \mathrm{y}$, empíricamente, la medida del $\angle A D B$ fue el argumento para verificar que $\triangle A D B$ es rectángulo en $D$ (Figura 10).

Figura 10 - Captura de pantalla de la representación dinámica elaborada por la pareja 1 como un medio para comprobar $m \angle A D B=90^{\circ}$.

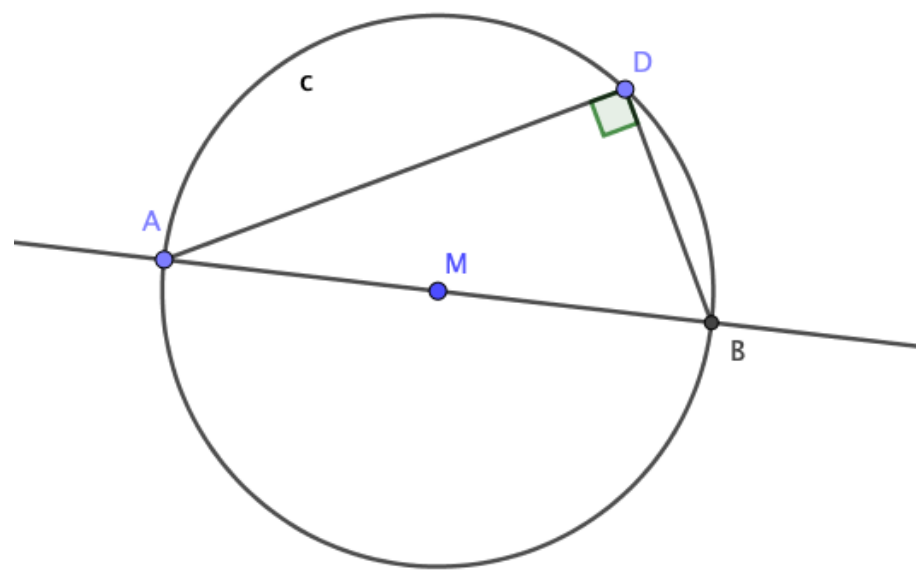

Fuente: Archivo GeoGebra elaborado por la pareja 1

Durante los acercamientos hacia la solución en el trabajo en plenaria, surgieron tres tipos de soluciones:

1. Geométrica: Identificaron que al $m \angle A D B$ como inscrito en una semicircunferencia por lo que $m \angle A D B=\frac{180^{\circ}}{2}=90^{\circ}$.

2. Algebraica: Basados en las propiedades de triángulos isósceles, medida de un ángulo central en una circunferencia y definición de circunferencia, construyeron un modelo algebraico, para demostrar que $m \angle A D B=90^{\circ}$.

3. Geométrica: Trazaron $\overleftrightarrow{D M}$ (ver Figura 10), identificaron la intersección de dicha recta con $c$ como el punto $G$ y construyeron el cuadrilátero $A D B G$, luego demostraron, que este es un rectángulo utilizando las propiedades de las diagonales de un rectángulo.

En este episodio, los estudiantes buscaron diferentes argumentos para justificar la solución del problema. En un primer acercamiento visual y empírico, definieron dos triángulos y un sector circular en GeoGebra para determinar el valor del área asociada a los casos favorables. Luego, construyeron un modelo algebraico basado en relaciones de la geometría euclidiana y de la trigonometría para obtener la totalidad de los casos favorables. El modelo algebraico como caso general de la solución fue validado al comparar sus resultados con la información generada por GeoGebra. De esta manera, los estudiantes tuvieron la oportunidad 
de relacionar contenidos de diferentes áreas de la matemática estudiadas, que generalmente, en los programas curriculares escolares se estudian por separado.

El uso GeoGebra proporcionó una base no solo para introducir y conectar acercamientos hacia soluciones integrando enfoques empíricos y formales, a través del uso de estrategias de arrastre de objetos, medición de áreas y ángulos y rastro de un punto con colores dinámicos dentro de la representación dinámica del problema.

\section{CONSIDERACIONES FINALES}

El uso de GeoGebra promueve una forma de razonar matemáticamente a partir de la exploración de familias de casos que resultan al arrastrar un punto dentro de la representación dinámica, y no solo de algunos casos como se haría con el uso de papel y lápiz. La medición de atributos de un objeto, en particular la medida de ángulos, en la exploración del problema puede conducir al estudiante a visualizar patrones en el comportamiento de objetos y formular conjeturas, y como consecuencia conectar conceptos de diversas áreas de la matemática y no verlos de forma aislada.

GeoGebra se convirtió en una herramienta que facilita el entendimiento o comprensión de las ideas matemáticas por parte de los estudiantes, como un proceso gradual. En específico, durante las dos sesiones de trabajo, los acercamientos iniciales en la resolución del problema fueron limitados, el uso sistemático de GeoGebra brindó a los futuros educadores matemáticos la oportunidad de adquirir y utilizar estrategias de arrastrar objetos dentro de la representación dinámica, medir los atributos de los objetos y observar el rastro de punto, con colores dinámicos, permitiéndoles diseñar un plan de solución, y refinarlo al presentar y discutir de manera abierta sus ideas con toda la clase.

Es importante resaltar que pensar un problema no solo implica hacerlo en términos de las posibilidades que ofrece GeoGebra, sino, el profesor debe incentivar a sus estudiantes a la búsqueda de formas de razonamiento y presentación de argumentos que involucren conceptos algebraicos o geométricos. En particular, los estudiantes encontraron en la representación gráfica del problema información visual y empírica para construir un modelo algebraico para justificar la solución.

En esta ruta, dentro del aula de matemáticas, es preciso que el profesor diseñe un espacio que favorezca el trabajo colaborativo entre los participantes durante la resolución de problemas. Es decir, debe orientar a los estudiantes hacia el desarrollo de ideas relacionadas con la práctica matemática, promover la comunicación entre pares y relacionar o conectar diferentes áreas del campo matemático; lejos de aplicar fórmulas o algoritmos para llegar a una solución.

Las dinámicas de interacción entre los estudiantes y su participación activa en las discusiones relacionadas con sus razonamientos, ya sea aquellos basados en argumentos visuales o empíricos producto del uso de GeoGebra hasta los algebraicos o geométricos, son elementos importantes en su proceso de aprendizaje, ya que sus ideas y sus formas de pensar se robustecen cuando son comunicadas a los demás.

\section{AGRADECIMIENTOS}

A la Escuela de Matemática de la Universidad de Costa Rica por el apoyo brindado. 


\section{REFERENCIAS}

GEERAERTS, L.; VENANT, F.; TANGUAY, D. Subterranean structures of technological tools and teaching issues in geometry. In: Proceedings of EDULEARN14 conference, 2014.

GROS, B.; KINSHUK; MAINA, M. Preface. In: GROS, B.; KINSHUK, M. (Org.), The future of ubiquitous learning. Learning design for emerging pedagogies, New York: Springer, 2016.

HEGEDUS, S. J.; MORENO-ARMELLA, L. Introduction: the trans-formative nature of 'dynamic' educational technology. ZDM - The International Journal on Mathematics Education, v. 41, n. 4, p. 397-398, 2009. ISSN 18639704. Disponível em: https://link.springer.com/article/10.1007/s11858-009-0201-9. Acesso em: 03 jun. 2020. Doi: https://doi.org/10.1007/s11858-009-0201-9

HEGEDUS, S. J.; TALL, D. O. Foundations for the future: The potential of multimodal technologies for learning mathematics. In: ENGLISH, L.; KIRSHNER, D. (Org.), Handbook of international research in mathematics education, 3rd ed., New York: Taylor \& Francis, 2016.

HÖLZL, R. Using Dynamic Geometry Software to Add Contrast to Geometric Situations - A Case Study. International Journal of Computers for Mathematical Learning, v. 6, n. 1, p.6386, 2001. ISSN 15731766. Disponível em: https://link.springer.com/article/10.1023/A:1011464425023. Acesso em: 15 jul. 2020. Doi: http://dx.doi.org/10.1023/A:1011464425023.

LESH, R.; ZAWOJEWSKI, J. S. Problem solving and modelling. In: LESTER, F. (Ed.), The second handbook of research on mathematics teaching and learning, NC: Information Age Publishing, 2007.

LEUNG, A.; BOLITE-FRANT, J. Designing mathematics tasks: The role of tools. In: WATSON, A.; OHTANI, M. (Org.), Task design in mathematics education, New ICMI Study Series. New York: Springer, 2015.

LEUNG, A.; BACCAGLINI-FRANK, A. Introduction. In: LEUNG, A.; BACCAGLINIFRANK, A. (Org.). Digital technologies in designing mathematic education tasks, Switzerland: Springer, 2017.

MASON, J.; JOHNSTON-WILDER, S. Designing and using mathematical tasks. St. Albans: Tarquin Publications, 2006.

NATIONAL COUNCIL OF TEACHERS OF MATHEMATICS. Principles and standards for school mathematics. Reston, VA: National Council of Teachers of Mathematics, 2000. 
NATIONAL COUNCIL OF TEACHERS OF MATHEMATICS. Focus in High School Mathematics: Reasoning and sense making. Reston, VA: National Council of Teachers of Mathematics. 2009.

PÓLYA, G. How to Solve it. Princeton: Princeton University Press. 1945.

PINHO, J. L. R.; MORETTI, M. T. Estimulando a criatividade em matemática em sala de aula através da formulação e resolução de problemas em geometria. REMATEC, v. 13, n. 28, p. 55-67, 2018. ISSN 1980-3141. Disponível em:

http://www.rematec.net.br/index.php/rematec/article/view/135. Acesso em: 19 jun. 2020.

POVEDA, W. Resolución de problemas matemáticos en GeoGebra. Revista do Instituto GeoGebra Internacional de São Paulo, v. 9, n. 1, p. 26-42, 2020. ISSN 2237-9657. Disponível em: https://revistas.pucsp.br/index.php/IGISP/article/view/46907. Acesso em: 18 jul. 2020. DOI: https://doi.org/10.23925/2237-9657.2020.v9i1p26-42.

POVEDA, W.; GOMEZ-ARCIGA, A. MOOC Resolución de problemas matemáticos y uso de tecnologías digitales: Su diseño e implementación. In: ALVARADO, A.; CARMONA, G.; MATA, A. (Org.) Una visión integradora. Tópicos Selectos de Educación en CITeM, México: Ecorfan, 2017. Disponível em:

https://www.ecorfan.org/actas/Una_vision_integradora/Una_visi\%C3\%B3n_integradora.pdf. Acesso em: 05 jun. 2020.

SANTOS-TRIGO, M.; CAMACHO-MACHÍN, M. Framing the use of computational technology in problem solving approaches. The Mathematics Enthusiast Journal, v. 10, n.1-2, p. 279-302, 2013. ISSN 1551-3440. Disponível em:

https://scholarworks.umt.edu/tme/vol10/iss1/. Acesso em: 06 jun. 2020.

SANTOS-TRIGO, M.; MORENO-ARMELLA, L.; CAMACHO-MACHÍN, M. Problem solving and the use of digital technologies within the Mathematical Working Space framework. ZDM: The International Journal on Mathematics Education, v. 48, n. 6, p. 827842, 2016. ISSN 18639704 Disponível em: https://link.springer.com/article/10.1007/s11858016-0757-0. Acesso em: 10 jun. 2020. DOI: https://doi.org/10.1007/s11858-016-0757-0.

SCHOENFELD, A. Mathematical problem Solving. New York: Academic Press. 1985.

SCHOENFELD, A. Method. In: LESTER, F. (Org.), Second Handbook of Research on Mathematics Teaching and Learning, New York: MacMillan, 2007. 
Submetido em: 19 de Outubro de 2020.

Aprovado em: 16 de Dezembro de 2020.

Publicado em: 18 de Janeiro de 2021.

\section{Como citar o artigo:}

POVEDA, W. E.; GARCÍA-CUÉLLAR, D. J. Estrategias asociadas al uso de GeoGebra en un contexto de resolución de problemas. Revista de Matemática, Ensino e Cultura REMATEC, Belém/PA, v. 16, n. 37, p. 61-79, Jan.-Abril, 2021. DOI: https://doi.org/10.37084/REMATEC.1980-3141.2021.n37.p61-80.id252 